\title{
Nepal Journal of Medical Sciences (NJMS)
}

\section{Author Guidelines}

\section{Introduction:}

Nepal Journal of Medical Sciences (NJMS) is the official journal of Manipal College of Medical Sciences (MCOMS), Pokhara, Nepal. It is a peer reviewed, open access, biomedical journal [ISSN 2091-1424 (Print) and 2091-1459 (Online at www.njmsmanipal.com)] and publishes the research based articles from the field of biomedical sciences which include Medicine, Surgery, Obstetrics and Gynaecology, Psychiatry, Paediatrics, Orthopaedics, Otorhinolaryngology, Ophthalmology, Anaesthesiology, Dermatology, Radiodiagnosis \& Radiotherapy, Dental, Forensic Medicine, Pathology, Anatomy, Biochemistry, Community Medicine, Microbiology, Pharmacology, Physiology and other allied biomedical disciplines. The journal is published twice a year (January and July). Each issue of NJMS publishes Original Articles, Review Articles, Case Reports, Editorials and Letters to the Editor.

\section{Manuscripts:}

Manuscripts must be prepared in accordance with the "Uniform Requirements for Manuscripts Submitted to Biomedical Journals" developed by the International Committee of Medical Journal Editors (http://www.icmje. org). Any manuscripts or substantial parts of it, submitted to the journal must not be under consideration by any other journal. In general, the manuscript should not have already been published in any journal or other citable form.

Please submit manuscript as word file and use American English throughout. Other required components and formatting:

1. Use 12 point font size (Times New Roman or Arial) and leave margins of $2.5 \mathrm{~cm}$. with double space throughout the manuscript.
2. Image formats accepted: JPEG, TIFF, GIF. Images should be of high resolution (300 dpi or higher).

3. Make three line tables which should be prepared without the use of tabs.

4. Check manuscript for spelling and grammar.

\section{Types of manuscripts:}

\section{Original articles}

Original articles are full-length reports of original research related to different disciplines of Medical Sciences. Articles cover topics relevant to innovative studies in the areas of interest. Original article submissions to NJMS cannot exceed 2500 words, excluding the abstract, figure and table legends, and references. The abstract should not exceed beyond 250 words.

\section{Review articles}

Scholarly evidence-based review articles will be considered for publication. Reviews must not exceed a total of 3,000 words, excluding the abstract, figure and table legends, and references. A maximum of 100 references are permitted. The abstract should not exceed beyond 250 words.

\section{Case reports}

Case reports include the interesting case reports with clinical significance or implications accompanied by the relevant literature review on those cases. They should not exceed a total of 1500 words, excluding references, figure legends or table legends. There should be no more than 15 references.

\section{Editorials}

Editorials express opinions on current topics of interest or provide comments on papers published elsewhere in the same issue. Editorials are usually solicited by the Editor. 


\section{Letters to the Editor}

Letters to the Editor should not contain material that has been submitted or published elsewhere. Letters in reference to a Journal article must not exceed 175 words (excluding references), and must be received within three weeks after publication of the article.

Letters not related to a Journal article must not exceed 400 words (excluding references). A letter can have no more than five references and one figure or table. A letter can be signed by no more than three authors.

\section{Cover Letter}

The cover letter must categorize the manuscript into respective discipline of Medical Sciences and mention the article type, which is appropriate for your article. Please write in a cover letter to explain why your manuscript should be published in the journal.

\section{Title Page}

Title limit: 120 characters with spaces

Short running title limit: 45 characters with spaces

Authors- Author name(s) with the highest academic degree of each author, name of the department and institution where work was performed and email addresses of each author.

Grant Support - List grant support and other assistance.

Correspondence - Provide name, complete mailing address, e-mail address, telephone number, and fax number of corresponding author.

Disclosures - All authors must disclose any potential conflicts (financial, professional, or personal) that are relevant to the manuscript. If the author(s) has nothing to disclose, this must be stated.

Writing Assistance - The names and funding source for individuals who provided writing assistance must be listed.

Author Contributions - List how each author was involved with the manuscript (eg, study concept and design; acquisition of data; analysis and interpretation of data; drafting of the manuscript; critical revision of the manuscript for important intellectual content; statistical analysis; obtained funding; administrative, technical, or material support; study supervision).

Keywords: 3-5 keywords; use key words from MeSH index- http://www.ncbi.nlm.nih.gov/mesh

Abstract: Abbreviations must be spelled out at least once. Do not use footnotes or references. Limit: 250 words. Organize according to the following headings: Background, Methods, Results and Conclusions.
Body of Paper: Body of paper includes Background, Methods, Results, Discussion and Conclusions.

Background: Describe the importance of the study and the precise research objective(s) or study question(s).

Methods: The methods section may employ subheadings at the discretion of the author.

- Design-describe the basic study design, e.g., randomized controlled trial, cross sectional study, cohort study, case series, survey, etc.

- $\quad$ Setting-specify whether the study was conducted in a primary or tertiary care setting, in an ambulatory care clinic or hospital, in the general community, etc.

- Participants-indicate the number of study subjects and how they were selected, recruited, and assigned to the intervention.

- Intervention-report the method of administration and duration of the intervention

Outline statistical methods used. When describing the results of hypothesis testing, report $\mathrm{P}$ values and/or confidence intervals; avoid using phrases such as "not significant."

Results: Provide the main outcomes of the study including confidence intervals or $\mathrm{P}$ values. Report the absolute values and risk differences so that readers can determine the absolute, as well as the relative, impact of the results. Discussion: Describe the innovative and important aspects of the study, but do not repeat in detail data or other information given in the Background or the Results section. Conclusion: State only conclusions that are linked with the goals of the study and directly supported by the evidence and the implications of the findings.

References: List references using superscripted Arabic numerals by order of appearance in the text. Cite personal communications and unpublished data directly in text without being numbered. All abbreviations should follow the Index Medicus abbreviations.

\section{Follow NJMS requirements for style:}

Article: (list first 3 authors followed by et al): 13. Uemura $\mathrm{N}$, Okamoto S, Yamamoto S, et al. Helicobacter pylori infection and the development of gastric cancer. $\mathrm{N}$ Engl $\mathrm{J}$ Med 2001; 345: 784-9.

Book: 18. Day RA. How to write and publish a scientific paper. Philadelphia: Institute for Scientific Information, 1979.

Article in Book: 22. Costa M, Furness JB, LlewellynSmith IF. Histochemistry of the enteric nervous system. In: Johnson LR, ed. Physiology of the gastrointestinal tract. 


\section{Author Guidelines}

Volume 1. 2nd ed. New York: Raven, 1987:1-40.

Note: Author submitting review article should include a section describing the methods used for locating, selecting, extracting, and synthesizing data. These methods should be summarized in the abstract.

\section{SUBMISSION CHECKLIST:}

Manuscripts submitted to NJMS should be double-spaced, accompanied by a cover letter and are divided into the following sections:

- Title page

- Abstract

- Body of paper (which includes Background, Methods, Results, Discussion and Conclusions)

- List of abbreviations used (if any)

- Conflicts of interests

- Authors' contributions
- Acknowledgments and Funding

- References

- $\quad$ Figure legends (if any)

- Tables and captions (if any)

- Description of additional data files (if any)

All pages of the manuscript must be numbered consecutively, starting with the title page as "page 1".

All manuscripts should be submitted electronically along with a cover letter signed by all authors to:

\section{Editor-in-Chief}

Umid K. Shrestha, MD, PhD

Email: editor@njmsmanipal.com

Nepal Journal of Medical Sciences (NJMS)

Manipal College of Medical Sciences

Pokhara, Nepal 